\title{
Mappings of Spaces with Families of Cones and Space-Time Transformations $(*)$.
}

\author{
A. D. ALEXANDROV (Novosibirsk)
}

To B. SEGRE on his seventieth birthday.

Summary. - Let $A, A^{\prime}$ be affine spaces with $\operatorname{dim} A \geqslant 3, \operatorname{dim} A=\infty$ being not excluded. Let $C, C^{\prime}$ be cones in $A$ and $A^{\prime} ; C_{x}, C_{y}^{\prime}$ denote the cones with the vertices $x, y$ got from $O, O^{\prime}$ by translation. Such one-to-one mappings $f: \mathcal{A} \rightarrow \mathcal{A}^{\prime}$ are considered that for every $x \in \mathcal{A}$ there exists such an $y \in A^{\prime}$ that $f\left(O_{x}\right)=C_{y}^{\prime}$. It is shown that $f$ is affine provided $O, O^{\prime}$ belong to one of certain classes of cones: those of the second order and «strictly convex》 ones, which are suitably defined, especially in infinitely dimensional space. Cones of the second order in n-space are determined by conditions of the following types: (1) $\varphi(x) \equiv$ $\equiv x_{1}^{2}+\ldots+x_{m}^{2}-x_{m+1}^{2}-\ldots-x_{n}^{2}=0, \quad(2) \varphi(x) \geqslant 0, \quad(3) \varphi(x)>0$ plus the vertex $(0, \ldots, 0)$. Strictly conven cones are of 6 types, the simplest representatives of which in 4 -spaces are 3 "dontble cones ": (1) $x^{2} \equiv x_{0}^{2}-\sum_{1}^{3} x_{i}^{2}=0$, (II) $x^{2} \geqslant 0$, (III) $x^{2}>0$ plus the vertex $(0, \ldots, 0)$, and three "ordinary" ones got from $(\mathrm{I})$-(III) by adding the condition $x_{0} \geqslant 0$. In space-time these cones correspond to different kinds of connection of events, e.g. (II) consists of points $x$ causally connected with $0=(0, \ldots, 0)$, an event at $x$ influences that at 0 or vice versa. Thus, the above result implies that a 1-1 mapping of space-time onto itself preserving causal connection is affine. Therefore mere preservation of causal connection implies Lorentz group.

\section{1. - Introduction.}

1.1. - Let $A$ be an affine space $\operatorname{dim} A=n+1 \geqslant 3$ and $x_{0}, x_{1}, \ldots, x_{n}$ cartesian co-ordinates in $A$. "Lorentz transformation with translation and dilatation" is a linear transformation preserving the form $x_{0}^{2}-\sum_{1}^{n} x_{i}^{2}$ plus parallel translation and multiplication of all $x_{i}$ by any number $\neq 0$. There takes place

Theonem $\mathrm{A}_{1}$. - Every one-to-one mapping of the space $A$ onto itself, which maps each cone

$$
\left(x_{0}-a_{0}\right)^{2}=\sum_{1}^{n}\left(x_{i}-a_{i}\right)^{2}
$$

onto a cone of the same kind, is a Lorentz transformation with translation and dilatation.

(*) Entrata in Redazione il 9 luglio 1974. 
(The essence of the result consists, of course, in the absence of any continuity hypotheses).

This theorem was established by me in 1949 [1]. It was formulated for fourdimensional space when (1) expressed the law of light propagation, and thus, as it was pointed out in [1], it follows from Theorem $A_{1}$ that constancy of light velocity alone implies Lorentz group with translations and dilatations.

In [1] there was given no proof but an indication of its basic point: "the linearity of the mapping is proved quite elementary by means of the well-known properties of the ruled surfaces of the second order (ordinary surfaces in three-dimensional space). The linearity being proved, the formulas of Lorentz transformation are deduced by an ordinary procedure". In the present paper we apply this elementary method to more general problems.

1.2. - Somewhat later the two other results have been obtained by me.

THEOREM $\mathrm{A}_{2}$. - Every one-to-one mapping of the space A onto itself which maps every cone

$$
x_{0}-a_{0}=\sqrt{\sum_{i=1}^{n}\left(x_{i}-a_{i}\right)^{2}}
$$

onto a cone of the same type is a Lorentz transformation with translation and dilatation.

THEOREM $\mathrm{A}_{3}$. - The same is true if we replace the cones (2) by solid cones

$$
x_{0}-a_{0} \geqslant \sqrt{\sum\left(x_{i}-a_{i}\right)^{2}} .
$$

(The roots being non-negative, (2) and (3) imply $x_{0} \geqslant a_{0}$ ).

The conditions of Theorems $A_{1}-A_{3}$ do not imply that vertices of cones are mapped into vertices. If $O_{a}$ denotes a cone indicated in one of our theorems, $a=\left(a_{0}, \ldots, a_{n}\right)$ being its vertex, and $f$ denotes the mapping, then our condition reads: for every $a \in A$ there exists such $a b=\left(b_{0}, \ldots, b_{n}\right)$ that $f\left(C_{a}\right)=C_{b}$, but we do not suppose $b=f(a)$.

Theorems $A_{1}-A_{3}$ were published in [2] with proofs (somewhat simplified by V. V. OvCHINNIKovA) and an explanation of their meaning for Relativity theory. In this theory $n=3$ and the cone (2) is the set of "world points " reached by light propagated from the world point $a=\left(a_{0}, \ldots, a_{3}\right)$; while the cone (3) is the set of all those world points which are reachable by any influence (transfer of the energyimpulse) from an event at $a$; in short, it is the domain of influence of an event at $a$. Thus, Theorem $A_{3}$ implies that every one-to-one mapping of space-time onto itself, which maps domains of influence onto domains of influence is Loretz transformation with translations and dilatation.

1.3. - Note a corollary of Theorem $A_{3}$. Let $Q_{\alpha}$ denote the interior of the cone (3), i.e. the set defined by the inequality

$$
x_{0}-a_{0}>\sqrt{\sum_{i=1}^{n}\left(x_{i}-a_{i}\right)^{2}} .
$$


THEOREM $\mathrm{A}_{4}$. - If a mapping $f: A \rightarrow A$ and its convers $f^{-1}$ map every $Q_{a}$ onto $a Q_{b}$, then $f$ is a Lorents transformation with translation and dilatation ( $\left.{ }^{1}\right)$.

Let $K_{a}$ denote the cone (3). It is evident that $K_{a}=\cap Q_{o}$ where all $Q_{c}$ with $a \in Q_{0}$ are involved. Hence

$$
f\left(K_{a}\right)=\bigcap_{a \in Q_{c}} f\left(Q_{c}\right)=\bigcap_{f(\alpha) \in Q_{b}} Q_{b}
$$

where all $Q_{b}$ with $f(a) \in Q_{b}$ are involved (as it follows from the condition that $f^{-1}$ maps every $Q_{b}$ onto a $\left.Q_{a}\right)$. Therefore, the set $\cap Q_{b}$ is $K_{y^{(a)}}$, and thus, $f\left(K_{a}\right)=K_{f(a)}$ q.e.d.

1.4. - Thet $O_{a}$ denote one of four sets (1)-(4); the relation $s \in C_{a}$ means a connection between the points $x$, a, defined by one of the formulas (1)-(4). According to the physical meaning of these formulas in Relativity theory we may say in the case of formula (1) that the points $a, x$ are connected by light, in the case of (2) $a$ acts upon $x$ by means of light (direct, non-reflected); in the case of inequality (3) the point $a$ causally precedes $x$, the event in $a$ acts (can act through other events) upon the event in $x$; in the case (4) an event in $a$ acts (can act) upon an event in $x$ "mechanically" (the action being transfered from $a$ to $x$ by a particle with nonzero mass $m_{0}$ ) or by reflected light.

From the view point of this physical interpretation it is quite natural to suppose that the mapping $f$ preserves the indicated connections between the points, i.e. $f$ preserves the relation $x \in C_{a}$ so that $x \in C_{a}$ implies $f(x) \in C_{f(a)}$. It is equivalent to the condition that the respective relation $(1),(2),(3)$ or $(4)$ for pairs of points $a, x$ is preserved under $f$ and $f^{-1}$. Therefore, Theorems $A_{1}$-A- imply

THEOREM A. - One-to-one mapping fof the space A onto itself proves to be a Lorentz transformation with translation and dilatation, as soon as $f$ and $f^{-1}$ preserve one of the relations (1)-(4); physically speaking, when $f$ and $f^{-1}$ preserve one of the following connections between the points (events):

(1) connection by direct light,

(2) influence by direct light,

(3) causal precedenee, influence (transfer of energy-impulse) of any kind,

(4) mechanical influence and the influence by indirect (reflected) light.

Case (3) seems to be most important, for it means, in short, that causality implies Lorentz group. In 1912 A. RoBb [8] gave an axiomatic of space-time based on the concept of causal order, but theorem $A(3)$ shows a fundamental role of the causality in the determination of the space-time structure in the most direct and short way.

(1) The condition on $f^{-1}$ may be omitted, as in Theorems $A_{1}-A_{3}$, but such a stronger form of Theorem $A_{4}$ does not follow from Theorem $A_{3}$ (or $A_{1}$ ) immediately. 
This meaning of theorem $A(3)$ and its consequences were expanded, in particular, in $[3-6]$.

1.5. - Theorem $A(1)$ or, what is the same, Theorem $A_{1}$ with the additional condition that the vertices of cones are mapped into the vertices was generalized by YU. BonIsov [8] in 1960; he proved:

THEOReM B. - One-to-one mapping f of the space A onto itself is affine as soon as $f$ and $f^{-1}$ preserve for every two points $x$, a, the relation

$$
\sum_{0}^{m-1}\left(x_{i}-a_{i}\right)^{2}-\sum_{m}^{n}\left(x_{i}-a_{i}\right)^{2}=0
$$

for a given $m, 0<m<n$; or in other words, if $f$ maps every cone with the eq. (4) onto a cone of the same type and the vertex into the vertex.

1.6. - Further in $\$ 2$ we prove a theorem which includes Theorem $\mathrm{A}$ and gives new physical results.

In $\S 3$ we give a summary of our further results which generalize Theorems $A_{1}-A_{3}$, $B$ in various aspects, in particular, for more general cones and for infinitely dimensional affine spaces. The proofs of these results are given in $\$ \$ 4-7$; in particular, $\$ 4$ contains an exposition of the method indicated in 1.1, based upon an application of ruled quadrics.

1.7. - During last ten years there appeared rather many papers on mappings of cones and analogous problems, e.g. [10-21]. However, the above quoted results obtained already long ago, as well as their physical consequences for relativity theory, have been left unnoticed and were partly discovered anew by some authors. Thus, it seemed useful to quote these old results the more so that, in their main part, as far as I knew, they were not rediscovered yet. In addition, their old proofs which we reproduce further are very simple, more simple than newly discovered ones.

For instance, in 1964 the paper by E. ZeEMas [10] "Causality implies Lorentz group " was published. which contained the cases (2), (4) of Theorem A but not Theorem $A_{3}$ or $A(3)$, i.e. there was no proof that the preservation of the causal precedence implies Lorentz group, so the statement in the title of the paper was left unproved though it has been known since 1953 [2].

In $1972 \mathrm{H}$. Borchers and G. HEGERFELDT [19] proved Theorem A(1). To stress the importance of this result they excalim (written in italies with exclamation mark): "Thus constancy of light velocity $c$ alone implies the Poincare group $\left(^{1}\right.$ ) (up to dilatations)!" Though, as was noted above, this result was published in [1] twenty two years earlier, then proved in [2] and after that was generalized by $\mathrm{YU}$. BoRIsov in his paper [8] which was reviewed in [8a] with a quotation of [2]. A related theorem on solid cones was proved in [7]. 


\section{2. - Space-time transformations.}

2.1. - Let again $A$ be an affine space, $\operatorname{dim} A \geqslant 3$, with co-ordinates $x_{0}, \ldots, x_{n}$. We denote by $x^{2}$ the quadratic form

$$
x^{2} \equiv x_{0}^{2}-\sum_{1}^{n} x_{i}^{2}
$$

For a pair of points $x, y$ we consider three symmetric relations:

$$
\begin{array}{lll}
\text { (I) }(x-y)^{2}=0, & \text { (II) }(x-y)^{2} \geqslant 0, & \text { (III) }(x-y)^{2}>0
\end{array}
$$

and three corresponding antisymmetric relations $\left(\mathrm{I}^{+}\right)-\left(\mathrm{III}^{+}\right)$got from (I)-(III) by adding the condition $x_{\theta} \leqslant y_{0}$.

THEOREM 1. - If a one-to-one mapping $f$ of the space $A$ onto itself and its conserve $f^{-1}$ preserves one of the six relations $(\mathrm{I})-\left(\mathrm{III}^{+}\right)$, then $f$ is a Lorentz transformation with translation and dilatation.

Thus, Theorem 1 comprises six cases and includes Theorem $A$, the relations (I), $\left(\mathrm{I}^{+}\right)-\left(\mathrm{III}^{+}\right)$being equivalent to (1)-(4) of $\S 1$. Case (II) is contained in a result got in [7], and case (III) - in [19]. Thus, all parts of Theorem 1 are not new, but we give them here a very simple proof.

Preservation of the antisymmetric relations $\left(\mathrm{I}^{+}\right)-\left(\mathrm{III}^{+}\right)$immediately implies that of symmetric relations (I)-(III) so that Theorem 1 for the relations $\left(\mathrm{I}^{+}\right)-\left(\mathrm{III}^{+}\right)$is but a simple corollary of it for the relations (I)-(III). We do not mention the relations $(x-y)^{2}<0,(x-y)^{2} \leqslant 0$ for, being opposite to (II) and (III), their preservation by $f$ and $f^{-1}$ is equivalent to that of (II) and (III).

2.2. - In order to prove Theorem 1 we reformulate it in terms of cones. We define the sets

(1) $C_{x}=\left\{y:(x-y)^{2}=0\right\}:$ a «superficial " cone with the vertex $x$;

(2) $K_{x}=\left\{y:(x-y)^{2} \geqslant 0\right\}:$ a "solid 》 cone;

(3) $Q_{x}=\{y:(x-y)>0\}$ : the interior of $K_{x}$.

Adding the condition $x_{0} \leqslant y_{0}$ we define the sets $\left(1^{+}\right)-\left(3^{+}\right): O_{x}^{+}, K_{w}^{+}, Q_{x}^{+}$- the halves of the sets $C_{x}, K_{x}, Q_{x}$.

The condition of Theorem 1 that $f$ and $f^{-1}$ preserve one of the relations (1)-(III $\left.{ }^{+}\right)$ is equivalent to the condition that for the corresponding set $M_{x}$ (i.e. $O_{x}$ or $K_{x}$ etc.), for every $x, f\left(M_{x}\right)=M_{f(x)}$. Thus, Theorem 1 is equivalent to the following.

THEOREM 1a. - If a one-to-one mapping $f$ of the space A onto itself maps every set $M_{\infty}$ of one of the above six types $(1)-\left(3^{+}\right)$onto the set $M_{f(x)}$, then $f$ is a Lorentz transformation with translation and dilatation. 
Further each time when a case of Theorem $1 a$ is considered $f$ denotes a mapping subject to the corresponding condition of Theorem $1 a$.

2.3. - Proof for the case $(I)-$ cone $C$.

2.3.1. - If $l$ is a generator of the cone $C_{a}, f(l)$ is that of the cone $f\left(C_{a}\right)=C_{f(a)}$.

We observe that if $b \in C_{a}$ and $b \neq a$, then $C_{a} \cap C_{b}$ is a generator $a b$ of $C_{a}$. Hence, owing to the conditions imposed on $f$, there follows our statement.

2.3.2. - If a two-plane $P$ contains two generators of a cone $C_{a}, f(P)$ is a twoplane too.

Proof. - Let $P$ be a plane of the indicated type. Then, for every $x \in P$ the set $P \cap C_{x}$ consists of two generators of $C_{x}$. Thus, $P$ is covered with two families of parallel generators of cones $C_{x}$.

As it is proved, $f$ maps generators onto generators. Therefore, the set $f(P)$ is a ruled surface with two families of straight generators, i.e. one of three surfaces: (1) a plane, (2) a hyperboloid, (3) a hyperbolic paraboloid.

But on the plane $P$ each generator crosses all the generators of the other family, while on a hyperboloid it does not take place. Therefore, $f(P)$ is not a hyperboloid.

On a paraboloid all generators of the same family are parallel to a two-plane. In our case they are generators of the cones $C_{x}$. But each cone has only two generators parallel to a given two-plane, and these generators belonging to different cones are pairwise parallel. Thus, there is only two direetions of generators parallel to a two-plane, and, therefore, $f(P)$ cannot be a paraboloid.

Hence, $f(P)$ is a plane, q.e.d.

2.3.3. - The mapping $f$ is affine.

Proof. - Let $l$ be a straight line. Talke a point $x \in l$ and two lines $l_{1}, l_{2}$ passing through $x$ within the cone $C_{x}$ in such a way that $l$ does not lie in the plane $\left(l_{1}, l_{2}\right)$, then either plane $P_{1}=\left(l, l_{1}\right), P_{2}=\left(l, l_{2}\right)$ contains two generators of the cone $C_{x}$.

Hence, owing to 2.3.2, $f\left(P_{1}\right), f\left(P_{2}\right)$ are planes, and as $l=P_{1} \cap P_{2}$, so $f(l)=$ $=f\left(P_{1}\right) \cap f\left(P_{2}\right)$ is a line.

Thus, $f$ maps every straight line onto a straight line, i.e. $f$ is affine.

2.3.4. - The mapping $f$ preserves the equality $(x-y)^{2}=0$ and is affine. Therefore, it differs from the transformation preserving the form $x^{2}=x_{0}^{2}-\sum x_{i}^{2}$, i.e. from a Lorentz transformation, by a translation and dilatation, q.e.d.

2.3.5. ReMark. - The proof of Theorem $A_{1}$ is almost the same. We observe that $C_{a} \cap C_{b}=C_{a} \cap O_{c}(a \neq b \neq c)$ if and only if $O_{a} \cap C_{b}$ is a generator of $C_{a}$. Therefore, if for every $x f\left(C_{x}\right)=C_{y}$, then generators are mapped onto generators, and it remains but to repeat the above deductions $2.3,2-2,3.4$. 
2.4. - Now Case (I) of Theorem 1 being considered, the proof for the remaining cases is very simple.

\subsection{1. - Proof for the Case $\left(\mathrm{II}^{+}\right)$.}

The following two observations are evident. If a point $x$ lies within a cone $K_{a}^{+}$, then there exist such points $y, z \in K_{a}^{+}$that $x \in K_{y}^{+} \cap K_{z}^{+}$and $K_{y}^{+} \not K_{z}^{+}, K_{z}^{+} \not K_{y}^{+}$. But if $x z_{a}$ lies on the boundary of $K_{a}^{+}$, i.e. $x \in C_{a}^{+}$, and $x \in K_{y}^{+} \cap K_{z}^{+}, y, z \in K_{a}^{+}$, then either $K_{y}^{+} \subset K_{z}^{+}$or $K_{z}^{+} \subset K_{y}^{+}$.

This difference of points $x$ lying within $K_{a}^{+}$or on the boundary is, evidently, preserved by a mapping $f$ which maps every $K_{x}^{+}$onto $K_{f^{\prime}(x)}^{+}$. Therefore, it maps a boundary onto boundary, i.e. $f\left(O_{x}^{+}\right)=C_{f(x)}^{+}$. Thus, we have the Case $\left(\mathrm{I}^{+}\right)$, and since it immediately follows from Case (I), Theorem 1 for the Case $\left(\mathrm{II}^{+}\right)$is proved.

2.4.2. - Proof for the Case $\left(\mathrm{III}^{+}\right)$. The following observation is obvious. For any given point $a$ the intersection of all $Q_{x}^{+}$containing $a$ is a cone $K_{a}^{+}$.

Therefore, if $f$ maps every $Q_{a}^{+}$onto $Q_{f(a)}^{+}$, it mpas every $K_{a}^{+}$onto $K_{f(a)}^{+}$. I.e. we have the above Case $\left(\mathrm{II}^{+}\right)$, and Theorem 1 in the Case $\left(\mathrm{III}^{+}\right)$is proved.

2.5. - Proof for the Cases (II), (III). We make the following evident observation. If two points $x, y$ lie in different "halves " of a cone $K_{a}$ (e.g. $x \in K_{a}^{+}, y \in K_{a}^{-}$), then $K_{x} \cup K_{y} \supset K_{a}$. But if $x, y$ lie in the same half (e.g. $x, y \in K_{a}^{+}$and $x, y \neq a$ ), then $K_{x} \cup K_{y} \not K_{a}$.

Hence, a mapping $f$ which maps every $K_{a}$ onto $K_{f(a)}$ maps halves onto halres, i.e. $f\left(K_{a}^{+}\right)$is either $K_{f(a)}^{+}$or $K_{f(a)}^{-}$.

And if for a given $a f\left(K_{a}^{+}\right)=K_{f(a)}^{+}$(or $K_{f(a)}^{-}$), so it is for all $a$ (since for every $K_{a}^{+}, K_{b}^{+}$there always exists $K_{c}^{+} \supset K_{a}^{+} \cup K_{b}^{+}$, but for $K_{a}^{+}, K_{b}^{-}$it is not so).

Thus, for every $x f\left(K_{x}^{+}\right)=K_{f(x)}^{+}$(or $\bar{K}_{f(x)}^{-}$), i.e. we have Case $\left(\mathrm{II}^{+}\right)$and Theorem 1 for the Case (II) is proved.

The above observation holds for the sets $Q_{a}$ : if $x \in Q_{a}^{+}, y \in Q_{a}^{-}$then $Q_{x} \cup Q_{y} \supset Q_{a}$, but if $x, y \in Q_{a}^{+}$(or $Q_{a}^{-}$), then $Q_{x} \cup Q_{y} \not \supset Q_{a}$. Therefore, above argument leads to the proof for the Case (III).

Thus, Theorem 1 is wholly proved.

2.6. - The physical meaning of Theorem 1 is clear from the interpretation given in 1.4. According to this interpretation the relations $\left(\mathrm{I}^{+}\right)-\left(\mathrm{III}{ }^{+}\right)$and (I)-(III) mean, respectively, influence and connection of events: (I) by direct light, (II) of any kind, (III) a mechanical one or by indirect light $\left({ }^{2}\right)$.

$\left({ }^{2}\right)$ The preservation of a relation being equivalont to that of an opposite one, we can replace $(x-y)^{2} \geqslant 0$ by $(x-y)^{2}<0$ which means that $x, y$ are not causally connected or are "possibly simultaneous". Thus, we may consider three pairwise disjunctive relations: (II) $(x-y)^{2}<0-$ no connection, (I) $(x-y)^{2}=0$ - direct light connection, (III) $(x-y)^{2}>0-$ other kind of connection.

Lorentz group with translations and dilatations is transitive on each of three sets of pairs $(x, y)$, defined by these relations. 
Theorem 1 asserts that the preservation of each of these six physical relations implies Lorentz group (with preservation of time direction in Cases $\left(\mathbf{I}^{+}\right)-\left(\mathbf{I I I} \mathbf{I}^{+}\right)$).

Case (II) is the most important; it means that the preservation of a general causal connection alone implies Lorentz group and thus determines the structure of space-time. The preservation of mechanical or light connection, as well as that of influence, is a physically more special and mathematically stronger condition.

We may say that the spatial-temporal structure of the world is its causal structure abstracted from its physical properties, except the mere relation of causal connection, in general. Thus, space-time may be defined as the set of all events with abstraction made of all properties with the above exception. The relation of influence $\left(\mathrm{II}^{+}\right)$determines, in addition, a direction of time.

This includes an answer to the problem set still by B. RiEmand: what is the basis of metric relations in space? We see that these metric relations are based upon causal connection of events.

2.7. - In [10] Theorem 1 is proved for Cases $\left(\mathrm{II}^{+}\right)$, ( $\left.\mathrm{III}^{+}\right)$, and the latter is interpreted as "causality implies Lorentz group ", i.e. causal precedence $x<y$ ("an event at $x$ can influence an event at $y$ " ) is defined by the relations $(x-y)^{2}>0, x_{0}<y_{0}$ and the group preserving it is called "causality group". Thus, the influence by direct light is excluded as if an event cannot influence the other one by direct light. This is the more strange and unsatisfactory that, if $(x-y)^{2}>0, x_{0}<y_{0}$ then an event at $x$ can influence an event at $y$ by the light scattered at some $z$. Then, according to the given definition we have to accept that $x \nless z$, though the influence from $x$ to $y$ is transfered through $z$. The only way to aroid such absurdities is to define causal precedence $x<y$ by the relations $(x-y)^{2} \geqslant 0, x_{0}<y_{0}$, that is by any kind of influence, i.e. by transfer of energy-impulse by mechanical particles, by light or in any some other way.

Thus, the statement that causality implies Lorentz group is proved in [1.0] at the cost of the very concept of causality in its ordinary general sense. But the statement that causality understood in the ordinary sense, without any restrictions, implies Lorentz group was proved in [2]. If instead of causal order we consider a symmetric relation of "undirected causality " implies Lorentz group too (with the reversal of time, of course).

2.8. - The fact that Cases (II), (II+) were left without consideration and, respectively, the restricted concept of causality with the exclusion of influence by light was introduced, is due to some difficulty that provide Cases (II), (II+) in comparison with the other ones.

We have shown in 1.3 that Case $\left(\mathrm{III}^{+}\right)$of Theorem 1 is a simple corollary of Case $\left(\mathrm{II}^{+}\right)$.

2.8.1. - Another reduction of $\left(\mathrm{III}^{+}\right)$to $\left(\mathrm{II}^{+}\right)$and $\left(\mathrm{I}^{+}\right)$is based on a definition of topology by the sets $Q^{+}$. These sets define the sets $Q_{a}^{-}=\left\{y: x \in Q_{y}^{+}\right\}$and the in- 
tersections $Q_{x}^{+} \cap Q_{y}^{-}$(provided $y \in Q_{x}^{+}$) make up an open basis in the space $A$. Therefore, if $f\left(Q_{x}^{+}\right)=Q_{f(x)}^{+}$for every $x$, then $f$ is continuous; hence

$$
f\left(K_{x}^{+}\right)=K_{f(x)}^{+}, \quad f\left(O_{x}^{+}\right)=O_{f(x)}^{+} .
$$

2.8.2. - The cones $K_{x}^{+}$(and the open cones $Q_{x}^{+} \cup(x)$ ) determine an order: $y \geqslant x$ if and only if $y \in K_{x}^{+}\left(y \in Q_{x}^{+}\right)$. Previous considerations can be expressed in terms of these orders. In particular, 2.8.1 offers a definition of topology by an order. If on a set an (partial) order $x \leqslant y$ is given, the sets $P_{x}^{+}=\{y: y \geqslant \infty\}, P_{x}^{-}=\{y: y \leqslant x\}$ are determined, and the simplest definition of topology is that of interval-topology: intervals $P_{x}^{+} \cap P_{y}^{-}\left(y \in P_{x}^{+}\right)$form an open basis. Thus, it is more convenient to suppose the sets $P_{x} \backslash(x)$ being open. Such a supposition is made, e.g. in a theory of "timelike spaces " [13] or "cinematies " [22]. Physically it means that the order $x \leqslant y$ is determined by a mechanical influence, influence by direct light being excluded. But in a theory that would correspond to a general concept of influence or causality the sets $P_{x}$ must be closed.

\section{3. - Further results.}

3.1. - Further we consider an affine space $A$ with $\operatorname{dim} A \geqslant 3$ not excepting $\operatorname{dim} A=\infty$. In this sense an affine space is a linear space over the field of real numbers, in which the translations $x \rightarrow x+a$ are introduced, and the points are considered as being "equivalent». Dimensionality $\operatorname{dim} A$ is understood as the greatest power of a set of independent vectors. An affine mapping $f: A \rightarrow A^{\prime}$ is a linear mapping up to a translation. A mapping $f: A \rightarrow A^{\prime}$ is affine provided it is one-to-one and maps straight lines onto straight lines.

3.2. - A cone is a set consisting of rays issued from a common point-the vertex of the cone. If the vertex $a$ is a centre of symmetry of the cone $C$, then $C$ consists of straight lines passing through $a$; in this case we call these lines generators of $C$ and $C$ a double cone. Otherwise, generators of a cone are rays issuing from the vertex, and the cone is called an ordinary one.

We call an ordinary cone $O$ strictly convex, $S O$-cone, provided it has the following properties.

(1) $C$ contains more than one generator;

(2) $P$ being a two-plane containing at least one generator of $C$, the set $V=P \cap C$ is an angle; a zero angle, i.e. a ray, not being excluded;

(3) the sides of the angle $V$ are "extremal generators", i.e. they are contained within no angle $V^{\prime}=C \cap P^{\prime}, P^{\prime}$ being a 2-plane (if $V$ is a ray, it is "the side " of $V$ ). sc-cone, thus defined, is convex. 
We define a "double SC-cone" and its extremal generators by the same conditions with the only difference that the set $V=P \cap C$ is a double angle, i.e. two vertical angles or a line. It is equivalent to the definition that a double SC-cone consists of two "halves "-ordinary $\$ O$-cone having a common vertex and being symmetric with respect to it.

$S C$-cone is contained in no plane (Suppose the contrary: $C \subset P$. Let $l$ be a nonextremal generator of $C$, and $Q$ is a two-plane: $l \subset Q \phi P$. Then $C \cap Q=l$ and, according to the definition of a $s C$-cone, $l$ must be an extremal generator).

Extremal generators of an $S C$-cone $K$ form a "superficial $S C$-cone" $\partial K$; all

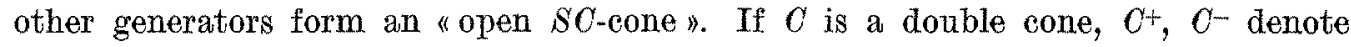
its halves. In order to distinguish an $S C$-cone from a superficial or an open one we call it a closed (solid) $S C$-cone.

3.3. - Thus, we consider six types of SC-cones, three double ones: (1) closed, (2) superficial, (3) open, and the same three types of ordinary cones. Six types of cones considered in $\S 2$ are the simplest instances of sC-cones.

If $K$ is a cone, $K_{x}$ denotes the cone with the vertex $x$, got from $K$ by translation.

There takes place the following theorem which generalizes Theorem 1 of $\S 2$.

THeorem 2. - Let $A, A^{\prime}$ be affine spaces and $K \subset A, K^{\prime} \subset A^{\prime}$ cones of one of the above six types. Let $f$ be sueh an one-to-one mapping of $A$ onto $A^{\prime}$ that for every $x \in A, f\left(K_{x}\right)=K_{f(x)}^{\prime}$. Then $f$ is affine.

The proof of this theorem is a mere repetition of that of Theorem 1.

Theorem 2 may be strenghened as follows.

THeORem 3. - Let in the conditions of Theorem $2 f$ be an one-to-one mapping of $A$ into $A^{\prime}$ and for every $x \in A, f\left(K_{x}\right)=K_{y}^{\prime}$. Than $f$ is affine and $f(A)=A^{\prime}$.

The condition $f\left(K_{x}\right)=K_{y}^{\prime}$ does not suppose $y=f(x)$; thus, Theorem 3 generalizes, in particular, Theorem $A_{1}-A_{3}$ of $\S 1$. The proof of Theorem 3 is given in $\$ \S 4,5$.

3.4. - Now we define cones of the second order in an affine space $A$ which is not supposed to be finite-dimensional.

An origin 0 being fixed, we may consider $A$ as a linear space; $x, a$ etc. will denote points as well as vectors. Let $A=A_{1} \oplus A_{2}$, so that every vector $x$ allows an unique representation:

$$
x=x_{1}+x_{2}, \quad x_{1} \in A_{1}, \quad x_{2} \in A_{2} .
$$

Let scalar products be defined in $A_{1}$ and $A_{2}$. We define a pseudo-scalar product in $A: x y=x_{1} y_{1}-x_{2} y_{2}$ and, respectively, $x^{2}=x_{1}^{2}-x_{2}^{2}$.

By full analogy with 2.2 we define the cones

(I) $C_{a}=\left\{x:(x-a)^{2}=0\right\}$

(II) $K_{a}=\left\{x:(x-a)^{2} \geqslant 0\right\}$

(III) $Q_{a}=\left\{x:(x-a)^{2}>0\right\} \cup(a)$. 
THEOREM 4. - Let in affine spaces $A, A^{\prime}$ pseudoscalar products be defined and, therefore, the sets (I)-(III), too. Let $f: A \rightarrow A^{\prime}$ be such an one-to-one mapping that for the sets $M \subset A, M^{\prime} \subset A^{\prime}$ of one of these three types for every $a \in A, f\left(M_{a}\right)=M_{b}^{\prime}$. Then $f$ is affine and $f(A)=A^{\prime}$.

The proof is given in $\$ 5,7$.

Theorem 4 includes, in particular, Borisov's Theorem $B$ of $\S 1$, being transformed. into it if the following conditions are added: (1) $A=A^{\prime}$, (2) $\operatorname{dim} A<\infty,(3) f(A)=A^{\prime}$, (4) $b=f(a)$, i.e. for every $a f\left(K_{a}\right)=K_{f(a)}$.

\subsection{Addendum to Theorem 4.}

3.5.1. - Apart from the above three kinds of sets (I)-(III), one may consider more 9 types: (1) 3 types of cones complementary to (I)-(III), i.e. the complements of the cones (I)-(III) plus the vertices, e.g. $(A \backslash K) \cup(a) ;(2) 6$ types of sets which are complements of the above 6 types of cones; these sets are the same cones without vertices, e.g. $C_{a} \backslash(a), A \backslash O_{a}$ etc. Theorem 4 implies the following.

CoRollary. - The conclusion of Theorem 4 remains valid if the sets $M, M^{\prime}$ belong to one of three types of complementary cones or to one of 6 types of complements of our cones provided, in the latter case, $f(A)=A^{\prime}$.

In partieular, a cone $M_{a}$ complementary to $Q_{\alpha}$ is $M_{a}=\left\{x:(x-a)^{2} \leqslant 0\right\}$. Thus it is the cone $K_{a}$ determined by the pseudoscalar product $x y=x_{2} y_{2}-x_{1} y_{1}=-x y$. Therefore, the statement of Corollary concerning the cones complementary to $Q_{a}$ coincides with the statement of Theorem 4 on the cones $K_{a}$. Analogously, the statement on cones complementary to $K_{a}$ coincides with that on cones $Q_{a}$.

3.5.2. - The space $A$ being infinite-dimensional, the question on the continuity of an affine mapping is not trivial one. Its solution for a mapping $f$ in Theorem 4 is as follows.

Introduce in $A$ a sealar product $(x y)=x_{1} y_{1}+x_{2} y_{2}$ and therefore the norm $|x|:|x|^{2}=x_{1}^{2}+x_{2}^{2}$. The following proposition takes place.

If one of subspaces $A_{1}, A_{2}$ is finite-dimensional, then every mapping $f$ subject to the conditions of Theorem 4 is continuous in the norm $|x|$. But if both $A_{1}, A_{2}$ are infinite-dimensional, then for every norm defined in $A$ there exists such $f: A \rightarrow A$ subject to the condition of Theorem 4 , which is not bounded in the given norm.

We shall leave this proposition as well as the above Corollary without proof which, by the way, is not difficult.

\subsection{Addendum to Theorem 3.}

3.6.1. - Along with six types of cones in Theorem 3 one may consider six types of complementary cones, i.e. the complement of a cone plus the vertex, e.g. the cone complementary to $K_{a}$ is $\left(A \backslash K_{a}\right) \cup(a)$. Then we may consider 12 types of complements to the above $6+6$ types of cones, i.e. the same cones without vertices. 
Altogether it makes up 18 types of sets. The following proposition takes place for them. The conclusion of Theorem 3 holds for these 18 types of sets, too.

We leave it here without proof.

3.6.2. - The problem on the continuity of a mapping $f$ in Theorem 3 has the following solution.

Let $Q_{x}^{+}, Q_{x}^{-}$denote the halves of an open double $S C$-cone (the vertex $x$ being excluded).

Consider the sets $Q_{x}^{+} \cap Q_{y}^{-}$, provided $y \in Q_{x}^{+}$, and define "Q-topology" in the space $A$ by accepting the collection of these sets for an open basis. Every type of cones - $C, C^{ \pm}, K$ etc. - that appear in Theorem 3 determines the corresponding sets $Q_{x}^{+}, Q_{x}^{-}$and, therefore, determines $Q$-topology in $A$. The following proposition takes place.

A mapping $\dot{f}$ in Theorem 3 is homeomorphic, in the sense of $Q$-topologies defined. in the spaces $A$ and $A^{\prime}$ by means of relative cones $M, M^{\prime}$.

The proof is obvious.

\section{4. - Lemmas.}

In this section the terms plane and mapping always denote a 2-plane and a oneto-one mapping.

4.1. Lemara. - Let a plane $P$ be covered with two families of parallel straight lines $l$ and let $g: P \rightarrow A^{\prime}$ be such a mapping that, for every $l, g(l)$ is a straight line. Then $g(P)$ is either a plane or a hyperbolic paraboloid.

Proof is contained in 2.3. - $g(P)$ is a ruled surface with two families of generators, i.e. it is either a plane or a paraboloid, or a hyperboloid. But hyperboloid is excluded, for on $P$ every two lines from different families cross each other while on a hyperboloid it is not so.

4.2. - Let in spaces $A, A^{\prime}$ double cones $C, C^{\prime}$ be given with the following properties:

(C) Every line passing through the vertex of the cone $C$ is contained in two planes, each of which crosses $C$ along (at least) two generators.

$\left(C^{\prime}\right)$ Generators of $C^{\prime}$ cover no plane angle.

It is easy to see that superficial $S C$-cones have these properties.

LeMma. - Let $C, C^{\prime}$ be the above cones and $f: A \rightarrow A^{\prime}$ such a mapping that the image of every line parallel to a generator of $O$ is a line parallel to a generator of $C^{\prime}$. Then $f$ is affine. 
The proof is contained in 2.3 ; at first, we note the following proposition.

4.2.1. - If the conditions of Lemma hold and a plane $P$ contains two lines parallel to generators of the cone $C$, then $f(P)$ is a plane.

This statement is quite analogous to 2.3 .2 and the proof is quite the same. The plane $P$ is covered by two families of lines parallel to generators of $C$ and their images are lines parallel to generators of $C^{r}$. Thus, owing to Lemma 4.1, $f(P)$ is either a plane or a paraboloic. On a paraboloid the generators belonging to the same family are parallel to a plane. In our case these generators being parallel to those of the cone $C^{\prime}$, it contradicts the condition $\left(C^{\prime}\right)$. Therefore, $f(P)$ is a plane.

4.2.2. - Now we finish the proof of Lemma. The condition $(C)$ implies that every line $l$ is the intersection of two planes, each of which contains two generators of the cone $C$. Hence, owing to 4.2.1 $f(l)$ is the intersection of two planes, i.e. $f(l)$ is a line and, therefore, $f$ is affine.

4.3. Lemma. - Let $C, C^{\prime}$ be ordinary cones with the properties $4.2(C),\left(C^{\prime}\right)$ and let a mapping $f: A \rightarrow A^{\prime}$ be such that the image of every ray parallel to a generator of $C$ is a ray parallel to a generator of $C^{\prime}$. Then $f$ is affine.

The proof is based on the following proposition which strengthens Lemma 4.1.

4.3.1. - Let $V$ be a (convex) angle in a plane and $g: V \rightarrow A^{\prime}$ such a mapping that every ray $l \subset V$ parallel to (or contained in) a side of $V$ is mapped onto a ray. Then $g(V)$ is either an angle on a plane or a part of a hyperbolic paraboloid bounded by two semigenerators.

Proof. - Let $l_{1}, l_{2}$ be the sides of the angle $V$. The said rays $l$ form two families of rays parallel to $l_{1}$ and $l_{2}$. Therefore, $g(V)$ proves to be a part of a ruled surface with two families of generators, i.e. $g(V)$ is a part either of a plane or of paraboloid, or of a hyperboloid. Since the origins of rays $l$ lie on the rays $l_{1}, l_{2}$, the origins of ray $g(l)$ lie on the rays $g\left(l_{1}\right), g\left(l_{2}\right)$; and every two rays with the origins on the different rays $g\left(l_{1}\right), g\left(l_{2}\right)$ cross each other.

This implies, at first, that $g(V)$ is a part of one of the above indicated. surfaces bounded by the rays $g\left(l_{1}\right), g\left(l_{2}\right)$, i.e. by semigenerators. And, secondly, a part of a hyperboloid is excluded, since on it not all the semigenerators eross each other, or not every point of every semigenerator $g(l)$ is a point of intersection with another semigenerator $g\left(l^{\prime}\right)$. This observation proves our statement 4.3.1.

4.3.2. - Let the condition of Lemma 4.3 hold and let $P$ be a plane parallel to two generators $l_{1}, l_{2}$ of the cone $C$. Then $P$ is mapped into a plane and, for every angle $V \subset P$ with the sides parallel to $l_{1}, l_{2}, f(V)$ is an angle.

Proof. - According to the conditions of Lemma the rays parallel to $l_{1}, l_{2}$ are mapped onto rays. Hence, applying 4.3.1, we conclude that the image of an angle $V$

16 - Annali di Matematica 
bounded by two such rays is an angle either on a plane or on a paraboloid. But the latter is excluded because of the condition $\left(C^{\prime}\right)$ like the proof of Lemma 4.2. Therefore, $f(V)$ is a plane angle, and, since it is true for every such angle $V \subset P$, so $f(P)$ is contained in a plane.

4.3.3. - Now we prove Lemma 4.3. Let $l$ be a line in $A$. According to the condition $(C)$ it is contained in two planes $P_{1}, P_{2}$, each of which is parallel to two generators of the cone $C$. According to $4.3 .2 f\left(P_{1}\right), f\left(P_{2}\right)$ are contained in some planes $Q_{1}, Q_{2}$, so that $f(l) \subset Q_{1} \cap Q_{2}$.

The planes $Q_{1}, Q_{2}$ do not coincide. For the proof we take a point $a \in l$. It is possible to construct on the planes $P_{1}, P_{2}$ such angles $V_{1}, V_{2}$ with the sides parallel to generators of the cone $C$ that $a$ lies within $V_{1}$ and $V_{2}$ : Then $f\left(V_{1}\right), f\left(V_{2}\right)$ are angles on the planes $Q_{1}, Q_{2}$, and the point $f(\alpha)$ lies within them. The rays parallel to the sides of $V_{1}$ and $V_{2}$ and covering these angles are mapped onto rays covering the angles $f\left(V_{1}\right), f\left(V_{2}\right)$.

Therefore, if the planes $Q_{1}, Q_{2}$ coincided, the angles $f\left(V_{1}\right), f\left(V_{2}\right)$ had common points which were not images of points belonging to the line $l=P_{1} \cap P_{2}$. Thus, the planes $Q_{1}, Q_{2}$ do not coincide, and, therefore, $f(l)$ is contained in a line.

Thus, every line is mapped into a line; it implies that $f$ is affine, and Lemma 4.3 is proved.

\section{5. - Proof of Theorem 3.}

Here we prove Theorem 3. It includes six statements concerning three types of both double and ordinary $S C$-cones defined in 3.2. We shall consider them successively starting with superficial cones. $f$ will denote such an one-to-one mapping $A \rightarrow A^{\prime}$ that for cones $M \in A, M^{\prime} \subset A^{\prime}$ of a type considered, for all $x \in A, f\left(M_{x}\right)=M_{y}^{\prime}$.

\subsection{Case I: double superficial $S C$-cone $C$.}

5.1.1. - Generators of $C$ are characterized by the following property.

If $l$ is a generator of $C_{a}$, there exist such cones $C_{b}, C_{c}(b \neq c)$ that $C_{a} \cap C_{b}=$ $=C_{a} \cap C_{c}=l$.

Conversely, if $b \neq c$ and $C_{a} \cap C_{b}=C_{a} \cap C_{c}$ then this intersection is a generator of $C_{a}$.

Proof. - Let $l$ be a generator of $O_{a}$. Then, as it is easy to see, $x \in l$ implies $C_{a} \cap C_{x}=l$. Therefore for every $b, c \in l$ we have $O_{a} \cap C_{b}=C_{a} \cap C_{c}=l$.

Let now $b \neq c$ and $C_{a} \cap C_{b}=C_{n} \cap C_{b}$ : The vertex of a double cone being its centre of symmetry, the set $M=C_{a} \cap O_{b}=C_{a} \cap C_{c}$ is symmetric with respect to the middle points of the segments $a b$, ac. 
The product of inversions at these points gives a translation which transforms $M$ into itself. Therefore, $M$ allows a group of translations. But, since $M$ is contained in a strictly convex cone $C_{a}$, it is possible only if $M$ is contained in a generator of $C_{a}$. But if $b \notin O_{a}, M=O_{a} \cap O_{b}$ is not contained in a generator. Therefore $b \in O_{a}$ and hence $M=C_{a} \cap C_{b}$ is a generator of $O_{a}$, q.e.d.

5.1.2. - A mapping $f$ that maps cones $O_{x}$ onto cones $O_{y}^{\prime}$ preserves, evidently, the above property which characterizes generators. Therefore, $f$ maps generators onto generators. Hence, owing to Lemma $4.2 f$ is affine.

5.2. - Case $\left(\mathrm{I}^{+}\right)$: ordinary superficial $B C$-cone $C^{+}$. We shall write $O$ instead of $C^{+}$.

5.2.1. - Generators of $O$ are charaeterized by the following property. (1) If $l$ is a generator of the cone $C_{a}$, there exist such cones $C_{b}, C_{c}\left(C_{b} \neq O_{b}\right)$ that $C_{a} \cap C_{b}=$ $=C_{a} \cap C_{c}=l$. (2) Conversely, if $C_{b} \neq O_{c}$ and $C_{a} \cap C_{b}=C_{a} \cap C_{c} \neq \emptyset$, then this intersection is a generator of $C$.

The statement (1) follows from the following observation. For every point $x$ lying on the prolongation of a generator $l$ of $C_{\alpha} C_{x} \cap C_{a}=l$.

Prove (2). Let $C_{a} \cap C_{b}=C_{a} \cap C_{c}=M \neq \emptyset$. Translating $O_{b}$ by the vector $b a$, we see that $C_{a}$ contains the set $M^{\prime}=M+b a$. Likewise, $C_{a} \supset M^{\prime \prime}=M+c a$.

Thus, $x$ and $y$ being two points of $M, O_{a}$ contains two triplets of points $x x^{\prime} x^{\prime \prime}$, $y y^{\prime} y^{\prime \prime}$, with $x x^{\prime}=y y^{\prime}=b a, x x^{\prime \prime}=y y^{\prime \prime}=c a$ (and, therefore, lying in a 3-plane). But the cone $C_{a}$, being strictly convex, can contain such triplets if and only if they are contained in one of its generators. Therefore, the vectors $b a$, $c a$ are parallel to this generator, hence, there follows the statement (2).

5.2.2. - A mapping $f$ that transforms the cones $C_{x}$ into the cones $O_{v}^{\prime}$ of the same type, preserves the property which, owing te 5.2.1, characterizes generators. Therefore it transforms generators into generators, and, owing to Lemma $4.3, f$ is affine.

5.3. - Case $\left(\mathrm{II}^{+}\right)$: ordinary $S C$-cone $K^{+}$. We shall write $K$ instead of $K^{+}$.

5.3.1. - A mapping $f$ that maps every $K_{a}$ onto $K_{b}^{\prime}$, maps vertices into vertices, i.e. $f\left(K_{a}\right)=K_{f(a)}^{\prime}$.

Proof. - Let $f\left(K_{a}\right)=K_{b}^{\prime}$.

Then we have such a point $c \in K_{a}$, that $f(c)=b$. Hence, $b \in f\left(K_{c}\right)$. But, $b$ being the vertex of $K_{b}^{t}=f\left(K_{a}\right)$, it implies that $f\left(K_{a}\right) \subset f\left(K_{c}\right)$. Thus, $K_{a} \subset K_{a}$. But, since $e \in K_{a}$, we have $K_{c} \subset K_{a}$. Therefore, $K_{c}=K_{a}$, i.e. $e=a$, i.e. $b=f(a)$.

5.3.2. - Now we may distinguish the interior and the boundary points of a cone $K_{a}$ as it was done in 2.4.1. Thus, we reduce Case (II+) to Case $\left(\mathrm{I}^{+}\right)$.

5.4. - Case (III'): ordinary open $S C$-cone $Q^{+}$. We write $Q, K$ instead of $Q^{+}, K^{+}$.

5.4.1. - A mapping $f$ that maps every $Q_{a}$ onto $Q_{b}^{\prime}$, maps vertices into vertices. The proof is quite the same as that of 5.3.1. 
5.4.2. - For every $a$ the cone $K_{a}$ is the intersection of all $Q_{x}$ which contain $a$ and are contained in any given $Q_{b}$ with $b \neq a \in Q_{b}$.

The proof is evident.

5.4.3. - For every a $f\left(\boldsymbol{K}_{a}\right)=K_{f(a)}^{\prime}$.

Proof. - Let $a \in Q_{b}, b \neq a$. Then $K_{a}=\cap Q_{x}$ where all $Q_{x}$ with $a \in Q_{x} \subset Q_{b}$ are involved. Therefore $f\left(K_{a}\right)=\cap f\left(Q_{x}\right)=\cap Q_{y}^{r}$.

Here $f(a) \in Q_{y}^{\prime}$, and, since $f\left(Q_{b}\right)=Q_{0}^{\prime}$, all such $Q_{y}^{\prime}$ that $y \in Q_{y}^{\prime}$ and $f(a) \in Q_{y}^{\prime}$ are involved. But because of 5.4 .2 it means that $\cap Q_{y}^{\prime}=K_{f(a)}^{\prime}$. Thus, $f\left(K_{a}\right)=K_{f(a)}^{\prime}$, and the Case $\left(\mathrm{III}^{+}\right)$is reduced to Case $\left(\mathrm{II}^{+}\right)$.

5.5. - Case (II): double closed $S C$-cone $K$.

5.5.1. - For any given point $a$

$$
(a)=\cap K_{x}
$$

where all such $K_{x}$ are involved that $a \in K_{x}, x \neq a$.

The proof is evident.

5.2.2. $-a \in K_{x}, x \neq a$, if and only if there exist such cones $K_{u}$ that $K_{u} \subset K_{a} \cup K_{x}$, $K_{w} \neq K_{a}, K_{x}$

Proof. - One can easily see that if $a \in K_{x}$, then for every point $u$ of the segment ax $K_{u} \subset K_{a} \cup K_{x}$.

Let $a \notin K_{x}$. Take a point $u$ and construct 2-plane $P \supset(a, x, u)$. The intersections $V_{a}=P \cap K_{a}, V_{x}=P \cap K_{x}, V_{u}=P \cap K_{u}$ are double angles with the parallel sides. Since $a \notin K_{x}$, the more $a \notin V_{x}$ and one can immediately see that $V_{u} \notin V_{a} \cup V_{x}$ for every position of $u$. Thus $K_{u} \notin K_{a} \cup K_{x}$ and 5.5.2 is proved.

5.5.3. - The vertex $a$ of a cone $K_{a}$ is the intersection of all such $K_{x}$ that there exist $K_{u} \subset K_{a} \cup K_{x}, K_{u} \neq K_{a}, K_{x}$.

This is an immediate corollary of 5.5.1 and 5.5.2.

5.5.4. - A mapping $f$ that maps every cone $K_{a}$ onto a cone $K_{b}^{\prime}$ maps vertices into vertices.

Proof. - Let $a$ be a given point and $f\left(K_{a}\right)=K_{b}^{\prime}$. Owing to 5.5.3

$$
(a)=\cap K_{x},
$$

where all such $K_{x}$ are involved that there exist $K_{u} \subset K_{a} \cup K_{x}, K_{u} \neq K_{a}, K_{x}$. 
Therefore $f(a)=\cap f\left(K_{x}\right), f\left(K_{x}\right) \cup f\left(K_{a}\right) \supset f\left(K_{u}\right)=K_{v}^{\prime}$. On the other hand, if we take all such $K_{y}^{\prime}$ that $K_{y}^{\prime} \cup f\left(K_{a}\right)$ contain $K_{w}^{\prime}$, then $\cap K_{v}^{\prime} \subset \cap f\left(K_{x}\right)$ and, because of 5.5 .3 $\cap K_{v}^{\prime}$ is the vertex of the cone $f\left(K_{a}\right)$, i.e. $\cap K_{y}^{\prime}=b$. Therefore $b=f(a)$, q.e.d.

5.5.5. - Now we can prove, repeating the argument 2.5, that halves of the cones $K_{x}$ are mapped onto halves of the cones $K_{v}^{\prime}$, and Case (II) is reduced to (II+).

5.6. - Case (III): double open $S C$-cone $Q$. This Case can be reduced to the Case $\left(\mathrm{III}^{+}\right)$by the same argument that has been just applied to the Case (II).

Thus, Theorem 3 is proved for all Cases (I)-(III+).

5.7. - Both Cases (II), (III) may be reduced to the Case (I) as follows. It is not difficult to verify two following propositions.

5.7.1. - Let $x \in K_{a}$, then $x \in C_{a}$ if and only if there exist such points $u \neq a, x$, that $K_{u} \supset K_{a} \cap K_{x}$.

5.7.2. - Let $x \notin Q_{a}$, then $x \in C_{a}$ if and only if there exist such points $u \neq a, x$ that $Q_{a} \backslash(u) \subset Q_{a} \cup Q_{x}$.

These propositions imply that if a mapping $f$ maps either cones $K_{a}$ onto cones $K_{f(a)}^{\prime}$ or cones $Q_{a}$ onto cones $Q_{f(a)}^{\prime}$, then in both cases it maps superficial cones $O_{a}$ onto $O_{f(a)}^{f}$, and, thus, the Cases (II), (III) are reduced to (I).

\section{6. - Proof of Theorem 4, Case (I).}

6.1. - Let the conditions of Theorem 4 hold. An origin being fixed in the space $A$, we equally speak on points $x$ and vectors $x$. We have $A=A_{1} \oplus A_{2}$, so for every vector $x, x=x_{1}+x_{2}, x_{1} \in A_{1}, x_{2} \in A_{2}$.

Further, if $a, x$ etc. are vectors, $a_{1}, a_{2}, x_{1}, x_{2}$ etc. are their components in $A_{1}$ and $A_{2}$.

Scalar products being defined in $A_{1}, A_{2}$, we have a pseudoscalar product in $A$ : $x y=x_{1} y_{1}-x_{2} y_{2}, x^{2}=x_{1}^{2}-x_{2}^{2}$.

We consider the cones $C_{a}=\left\{x:(x-a)^{2}=0\right\}$ and we put $\left\{x: x^{2}=0\right\}=C$.

Let in a space $A^{\prime}$ cones $C_{b}^{\prime}$ be defined in an analogous manner, and let a one-toone mapping $f: A \rightarrow A^{\prime}$ be such that for every $a \in A$

$$
f\left(C_{a}\right)=C_{b}^{\prime} .
$$

Theorem 4 asserts that $f$ is an affine mapping of $A$ onto $A^{\prime}$. In this paragraph we prove this statement.

6.2. - On planes contained in a cone. Planes of any dimensionality $\geqslant 1$ are meant. When saying that a plane $P$ is contained in a cone $O_{a}: P \subset O_{a}$, we imply that it passes through its vertex. One-dimensional plane $P \subset C$ is a generator of $C$. 
6.2.1. - The plane $(a, b)$ stretehed on vectors $a, b$ is contained in $O$ if and only if

$$
a^{2}=b^{2}=a b=0 .
$$

Proof. - The plane $(a, b)$ being composed by vectors $(\alpha a+\beta b)$ it is contained in $O$ if and only if $(\alpha a+\beta b)^{2}=0$ for every $\alpha, \beta$. This is equivalent to (1).

6.2.2. - If $A_{1}$ or $A_{2}$ is one-dimensional, $C$ contains no planes but generators. But if $\operatorname{dim} A_{1}$ and $\operatorname{dim} A_{2}>1, O$ contains 2-planes.

Proor. - Let $\operatorname{dim} A_{1}=1$, i.e. $A_{1}$ be a line. Then, if $R$ is a three-plane and $A_{1} \subset R$, the set $R \cap C$ is a cone of revolution. Therefore, $C$ contains no planes but generators (for, otherwise, we could draw $R$ through 2-plane $P \subset C$ and would have $P \subset R \cap O)$.

Let now $\operatorname{dim} A_{1}, \operatorname{dim} A_{2}>1$. Take independent vectors $a_{1}, b_{1} \in A_{1}$ and such vectors $a_{2}, b_{2} \in A_{2}$ that

$$
a_{1}^{2}=a_{2}^{2}, \quad b_{1}^{2}=b_{2}^{2}, \quad a_{1} b_{1}=a_{2} b_{2} .
$$

Then the vectors $a=a_{1}+a_{2}, b=b_{1}+b_{2}$ satisfy (1) and, therefore, $(a, b) \subset C\left(^{3}\right)$.

6.2.3. - If vectors $a, b \in C$ are independent, there exists such a vector $c \in C$ that $(a, c) \subset C$ but $(b, c) \notin C$.

Proof. - If $(a, b) \notin C$ the statement is obvious, for then every plane $P \subset C$ containing $a$ does not contain $b$.

Let $(a, b) \subset C$, and therefore (1) takes place. Define a vector $c$ with the components:

$$
c_{1}=\frac{2 a_{1} b_{1}}{a_{1}^{2}} a_{1}-b_{1}, \quad c_{2}=b_{2} .
$$

Then, using (1), a simple calculation gives $e^{2}=a c=0$, and since $a^{2}=0$, we have $(a, c) \subset C$.

On the other hand, owing to $b_{1}^{2}=b_{2}^{2}$, we have:

$$
b e=b_{1} e_{1}-b_{2} c_{2}=\frac{2}{a_{1}^{2}}\left(\left(a_{1} b_{1}\right)^{2}-a_{1}^{2} b_{1}^{2}\right) .
$$

Hence $b c \leqslant 0$. Suppose $b c=0$, i.e. $\left(a_{1} b_{1}\right)^{2}=a_{1}^{2} b_{1}^{2}$. Then (1) implies $\left(a_{2} b_{2}\right)^{2}=a_{2}^{2} b_{2}^{2}$. Therefore, $b_{1}=\lambda a_{1}, b_{2}=\mu a_{2}$, and owing to (1) $\lambda=\mu$, i.e. $b=\lambda a$. Therefore, $a, b$ being independent, $b c \neq 0$ and thus $(b, c) \not \subset C$, q.e.d.

$\left.{ }^{3}\right)$ ReMaARK. - Let $P_{1}$ be a subspace $P_{1} \subset A_{1}$, and $\varphi$ a mapping $P_{1} \rightarrow A_{2}$ preserving scalar product. Then the set $P$ of all $x$ with $x_{2}=\varphi\left(x_{1}\right), x_{1} \in P_{1}$, is a plane contained in $O$. Conversely, let $P C O$ be a plane and $P_{1}$ a set of all components $x_{1}$ of vectors $x \in P$. Define a mapping $\varphi: P_{1} \rightarrow A_{2}$ as follows, $\varphi\left(x_{1}\right)=x_{2}$, where $x_{2} \in A_{2}$ and $x=x_{1}+x_{2} \in P$. This mapping $\varphi$ preserves scalar product. 
6.2.4. - We say that a plane $P \subset C$ is a maximal one, if there exists no plane $P^{\prime} \subset C$ such that $P \subset P^{\prime}, P \neq P^{\prime}$. Every plane $P \subset C$ is contained in a maximal one.

LEMra. - A generator of the cone $O$ is the interseetion of all maximal planes containing it.

Proof. - Let $l$ be a generator of $C$ and $M$ the intersection of all maximal planes $P \supset l$. In order to prove that $M=l$, it suffice to show that $b \in C \backslash l$ implies $b \notin M$.

Let $a \in l, b \in O \backslash l(a, b \neq 0)$. Then $a, b$ are independent and owing to 6.2.3 there exists such $c \in O$ that $(a, c) \subset C,(b, e) \not C$. Therefore, no plane $P \subset O$ containing $(a, c)$ can contain $b$. Hence, $b \notin M$, and our Lemma is proved.

6.2.5 - Let $a$ be such a vector that $a^{2} \neq 0$. The mapping $x \rightarrow x^{\prime}$ :

$$
x^{\prime}=x-2 a \frac{a x}{a^{2}}
$$

is isometric (i.e. $x^{\prime} y^{\prime}=x y$ ) and, therefore, transforms the cone $C$ into itself. This mapping is the "reflection " in the plane $a x=0$.

Every two generators $l, l_{1}$ which lie in no plane $P \subset C$, could be transormed one into another by means of such a reflection.

Proor. - Let $b \in l, b_{1} \in l_{1}\left(b, b_{1} \neq 0\right)$. Then $\left(b-b_{1}\right)^{2} \neq 0$ (since, if $b, b_{1} \in C$, then $b^{2}=b_{1}^{2}=0$, and $\left(b-b_{1}\right)^{2}=0$ implies $b b_{1}=0$, thus, owing to $6.2 .3, b, b_{1}$ lie in a plane $P \subset C$ ). Put in (2) $a=b-b_{1}$. Because of $b^{2}=b_{1}^{2}=0$ we have $\left(b-b_{1}\right)^{2}=-2 b b_{1}$ and, therefore, (2) for $a=b-b_{1}$ and $x=b$ gives:

$$
b^{\prime}=b+2\left(b-b_{1}\right) \frac{\left(b-b_{1}\right) b}{2 b b_{1}}=b-\left(b \geqslant b_{1}\right)=b_{1},
$$

q.e.d.

6.3. - On intersections of cones.

6.3.1. - Let $a \in C(a \neq 0)$, then $C_{a} \cap C$ is the sum of all planes $P \subset C$ which contain $a$, i.e. which contain the generator $l \ni a$.

Proor. - Let $M$ be the said sum. If a plane $P \subset C$ and $a \in P$, then $P \subset C_{a}$. Thus, $P \subset C \cap C_{a}$, and therefore $M \subset C \cap C_{a}$.

Let now $b \in C \cap C_{a}$. Then $b^{2}=(b-a)^{2}=0$ and, because of $a^{2}=0, a b=0$. Therefore, owing to 6.2.1, $(a, b) \subset C$. Since that is true for every $b \in C \cap C_{a}$, we conclude that $C \cap C_{a} \subset M$. Thus $C \cap C_{a}=M$, q.e.d.

6.3.2. LemMA. - Let $a, b$ be two different points. Then $C \cap C_{a}=C \cap C_{b}$ if and only if $a, b$ lie on a generator of the cone $O$ (not in its vertex). 
Proof. - (1) If $a, b$ lie on a generator of $C$ (not in the vertex), then $C \cap C_{a}=C \cap C_{b}$. It immediately follows from 6.3.1.

Now we shall prove: (2) if $C \cap C_{a}=C \cap C_{b}$, then the points $a, b$ belong to $a$ generator of $C$.

Let one of the points $a, b$ belong to $C$ : e.g. $a \in C$. Then, owing to 6.3.1, $C \cap C_{a}$ is the sum $M$ of all maximal planes $P: a \in P \in C$. On the other hand, owing to 6.2.7, the intersection of these planes is generator $l_{a}$ of $C, a \in l_{a}$.

But $a \in C$ implies $0 \in C_{a}$ and, since $O \cap C_{a}=C \cap C_{b}, 0 \in C_{b}$ too; hence $b \in C$. Therefore, the above observation is applied to $b$ : the maximal planes $P$ that form the set $M$ contain $b$ and their intersection is the generator $l_{b}$. Thus, $l_{a}=l_{b}$, i.e. $a, b$ belong to one generator of $C$.

Let now $a, b \notin C$. The set $C \cap O_{a}$ is defined by the equations $x^{2}=0,(x-a)^{2}=0$, or $x^{2}=0,2 a x=a^{2}$. It means that $C \cap O_{a}=C \cap P_{a}, P_{a}$ being the plane $2 a x=a^{2}$. Likewise, $O \cap C_{b}=C \cap P_{b}, P_{b}$ being the plane $2 b x=b^{2}$.

Thus, $O \cap C_{a}=C \cap C_{b}$ implies $C \cap P_{a}=C \cap P_{b}$ : But, since $a \neq b, P_{a} \neq P_{b}$ and the above equality implies that $O \cap P_{a}$ is contained in the plane $Q=P_{a} \cap P_{b}$ with $\operatorname{codim} Q=2$. But it is impossible. In fact, let $e \in P \backslash Q$. Let $R$ be a 3-plane containing components $c_{1}, c_{2}$ and a vector $d \in A_{2}$ independent with $c_{2}$ : Then the set $C^{\prime}=C \cap R$ is a cone of revolution in $R$ with the axis along $c_{1}$; the set $P^{\prime}=P_{a} \cap R$ is a plane and $Q^{\prime}=Q \cap R-$ a line. Therefore, $C^{\prime} \cap P^{\prime} \neq C^{\prime} \cap Q^{\prime}$, hence $O \cap P_{a} \neq C \cap Q$.

Thus, if $a, b \notin O$, the equality $C \cap C_{a}=C \cap C_{b}$ is impossible, and our Lemma is proved.

\subsection{3 - Lemma 6.3.2 implies:}

A generator of $C$ (the vertex being excluded) is the set of all such $a \in C$ for which there exist such $b \neq a$ that $C \cap O_{a}=C \cap O_{b}$.

6.3.4. - The vertex of the cone $C$ is characterized by the following property. $A$ point $z \in C$ is the vertex of $C$ if and only if for every cone $C_{a} \neq C$ containing $z$ there exist such cones $C_{b} \neq C_{a}$ that $O \cap C_{a}=C \cap C_{b}$.

Proof. - Let $z$ be the vertex of $C$ and $z \in C_{a}(z \neq a)$. Then $a \in C$, too, and owing to 6.3 .2 , for every $b$ lying on the same generator, $O \cap C_{a}=O \cap C_{b}$.

Let now $z$ be different from the vertex of $C$. Take a point $a \in C_{z} \backslash C$. Then $z \in O_{a}$.

Suppose $C \cap C_{a}=C \cap C_{b}$. Then, owing to Lemma 6.3 .2 , the points $a, b$ belong to a generator of $C$, i.e. $a \in C$ in contradiction with the choice of $a$. Therefore, for every $b \neq a, C \cap C_{a} \neq C \cap C_{b}$.

\section{4. - Proof of Theorem 4. Case (I).}

Now we turn to a mapping $f: A \rightarrow A^{t}, f\left(O_{o}\right)=C_{b}^{\prime}$ for every $a \in A$, 
6.4.1. - For every $a, f\left(C_{a}\right)=C_{f(a)}^{\prime}$. It is an immediate corollary of 6.3 .4 for $f$ preserves the property which, owing to 6.3.4, characterizes the vertex of a cone.

6.4.2. $-f(A)=A^{\prime}$ and for every $b \in A^{\prime}, f^{-1}\left(O_{b}^{\prime}\right)=O_{a}, a=f^{-1}(b)$.

Proof. - For every $y \in C^{\prime}=f(O)$ there exists $x=f^{-1}(y) \in O$, and, thus, owing to 6.4.1, $C_{y}^{\prime}=f\left(C_{x}\right)$. But the sum of all $C_{y}^{\prime}$ with $y \in C^{\prime}$ is $A^{\prime}$. Hence, $f(A)=A^{\prime}$.

Therefore, for every $y \in A^{\prime}$ we have $f^{-1}(y)=x$ and, owing to $6.4 .1, f\left(C_{x}\right)=C_{y}^{\prime}$, i.e. $f^{-1}\left(C_{y}^{\prime}\right)=C_{x}$.

6.4.3. - $f$ maps generators onto generators.

It is an immediate corollary of 6.3 .3 and 6.4 .1 , for $f$, mapping vertices into vertices, preserves the property that, by 6.3 .3 , characterizes generators.

6.4.4. - For every plane $P \subset O, f(P)$ is a plane and, $P$ being a maximal one, $f(P)$ is either; moreover, if $P$ is not a line, $f$ is affine on $P$.

Proof. - Every line $l$ in a plane $P \subset C$ is a generator of every cone $C_{a}$ with $a \in l$. Thus, since $f$ maps generators onto generators, $f(P)$ is a plane and $f$ is affine on $P$, provided $P$ is not a line.

Let a plane $P^{\prime} \subset C^{\prime}$ contain $f(P)$. Then $f^{-1}\left(P^{\prime}\right)$ is a plane and $f^{-1}\left(P^{\prime}\right) \supset P$. Therefore, if $\boldsymbol{P}$ is a maximal plane $f^{-1}\left(\boldsymbol{P}^{\prime}\right)=P$, i.e. $\boldsymbol{P}^{\prime}=f(\boldsymbol{P})$. Hence $f(\boldsymbol{P})$ is a maximal plane, too.

6.4.5. - If $\operatorname{dim} A_{1}=1$ (or $\left.\operatorname{dim} A_{2}=1\right), f$ is affine.

Proof, - If $\operatorname{dim} A_{1}=1$, generators of $C$ are maximal planes (by 6.2.2) and 6.4.3, 6.4.4 imply that $f(l)$ are generators and maximal planes of the cone $C^{\prime}=f(C)$. Hence, $C^{\prime}$ does not contain planes but generators. Thus, since generators are mapped onto generators, Iumma 4.2 is applicable, and $f$ is affine.

6.5. - Further we suppose that $O$ contains 2-planes.

6.5.1. - Let $P_{0}, P_{1} \subset C$ be such maximal planes, that the plane $Q_{0}=P_{0} \cap P_{1}$ has codimensionality 1 in them. Then $R$ being the plane stretched on $P_{0}$ and $P_{1}, f(R)$ is one stretched on $f\left(P_{0}\right)$ and $f\left(P_{1}\right)$.

Before proving this statement we make two remarks.

(1) The said pairs of planes $P_{0}, P_{1}$ exist: for every maximal plane $P_{0} \subset O$ there exist such maximal planes $P_{1} \subset O$ that $P_{0} \cap P_{1}$ has in $P_{0}$ and $P_{1}$ codimensionality 1 .

Such $P_{1}$ may be got by means of pseudoreflection of $P_{0}$ at any plane $a x=0$ (provided it does not contain $P_{0}$ ). 
(2) If $C$ contains no planes but generators, our statement 6.5.1 is reduced to that a 2-plane containing two generators of $C$ is transformed into a 2-plane. Thus, 6.5.1 is a generalization of 4.2 .1 .

6.5.2. - Proof of 6.5.1. Let $P, P^{\prime}, Q$ denote planes contained in $R$ and parallel to $P_{0}, P_{1}, Q_{0}$, respectively. For every $P$ and $P^{\prime}$ the intersection $P \cap P^{\prime}$ is $Q$.

Each plane $\left(P^{\prime}\right)$ is contained in every cone $C_{x}$ with $x \in P\left(x \in P^{\prime}\right)$. Hence, owing to 6.4.4, $f$ is affine on each $P$ and $P^{\prime}$. In particular, $f(P), f\left(P^{\prime}\right), f(Q)$ are planes $f(Q)=f(P) \cap f\left(P^{\prime}\right)$ and $f(Q)$ has codimensionality 1 in $f(P)$ and $f\left(P^{\prime}\right)$.

We prove that all $f(Q)$ are parallel to each other. Let $Q_{1}, Q_{2}$ be two of the planes $Q$. If they are contained in $P$ (or $\left.P^{\prime}\right), f\left(Q_{1}\right)$ and $f\left(Q_{2}\right)$ are parallel owing to affinity of $f$ on $P$. Suppose $Q_{1}, Q_{2}$ are contained neither in $P$, nor in $P$. Take such $P, P^{\prime}$ that $P \supset Q_{1}, P^{\prime} \supset Q_{2}$. Then $P \cap P^{\prime}=Q_{3}$, and, by previous argument, $f\left(Q_{1}\right) \| f\left(Q_{3}\right)$, $f\left(Q_{2}\right) \| f\left(Q_{3}\right)$, hence $f\left(Q_{1}\right) \| f\left(Q_{2}\right)$.

Thus, $f(R)$ consists of parallel planes $f(Q)$ as $R$ consists of planes $Q$.

6.5.3. - Now we shall prove that $f(R)$ is a plane.

Let $S$ be such a 2-plane that $S \subset R$ and $S \cap Q_{0}=(0), 0$ being the vertex of $C$. Then $R$ is stretched on $S$ and $Q_{0}$, and every plane $Q$ crosses $S$ at a single point. The intersections $P_{0} \cap S=l_{0}, P_{1} \cap S=l_{1}$ are generators of $C$, which are not contained in $Q_{0}$. Respectively, for every $P$ and $P^{\prime}$ the intersections $P \cap S=l, P^{\prime} \cap S=l^{\prime}$ are generators, $l\left\|l_{0}, l^{\prime}\right\| l_{1}$ (of every cone $C_{x}$ with $x \in l$ or $x \in l^{\prime}$ ). Thus, $\&$ is covered with two families of parallel generators $l, l$.

Hence, by Lemma 4.1, $f(S)$ is either a plane or a hyperbolic paraboloid.

In the first case, $f(R)$ consists of parallel planes $f(Q)$ which pass through the points of the plane $f(S)$, and therefore $f(R)$ is a plane.

Let now $f\left(S^{\prime}\right)$ be a paraboloid. Its generators are the lines $f(l)$ and $f\left(l^{\prime}\right)$, the first belonging to one family and the second to the other one. Hence, the lines $f(l)$ are parallel to a 2-plane $T$. We take $T$ containing the vertex of $C$, so that $T \supset f\left(l_{0}\right)$.

Since the generator $l_{0}$ is not contained in the plane $Q_{0}, f\left(l_{0}\right) \notin f\left(Q_{0}\right)$. And since $T \supset f\left(l_{0}\right), f\left(Q_{0}\right) \not T$. Therefore, there are two possible cases: (1) $T \cap f\left(Q_{0}\right)$ is a line, (2) $T \cap f\left(Q_{0}\right)$ is a point.

Let $V$ be the plane stretched on $f\left(Q_{0}\right)$ and $T$. Since $T \supset f\left(l_{0}\right)$ and the plane $f\left(P_{0}\right)$ is stretched on $f\left(Q_{0}\right)$ and $f\left(l_{0}\right), V \supset f\left(P_{0}\right)$. In the case (1) $V=f\left(P_{0}\right)$, in the case (2) $V \neq f\left(P_{0}\right)$.

Every plane $P$ consists of planes $Q$ crossing the line $l \subset P$. Respectively, $f(P)$ consists of planes $f(Q)$ crossing the line $f(l)$. And since $f(Q) \| f\left(Q_{0}\right)$ and $f(l) \| T$, so $f(P) \| V$.

Let $V=f\left(P_{0}\right)$. Then $f(P) \| V$ implies that the planes $f(P)$ are parallel to $f\left(P_{0}\right)$. They cross the line $f\left(l_{0}\right)$ and through each point $x \in f\left(l_{0}\right)$ passes one of them. Therefore, their sum $\cup f(P)$ is a plane. But, since the plane $R$ consists of the planes $P$, i.e. $R=\cup P$, so $\cup f(P)=f(R)$ and, therefore, $f(R)$ is a plane.

Suppose now $V \neq f\left(P_{0}\right)$. As it is proved, the planes $f(P)$ are parallel to $V$. We translate them so they pass through the point 0 . With them the lines $f(l)$, i.e. gener. 
ators of paraboloid $f(S)$ are translated twoo. Being parallel to the plane $T$, they will lie in it, covering it with the exception of a line. The plane $V$ being stretched on $T$ and $P_{0}$, the translated planes $f(P)$ fill the plane $V$ with the exception of a plane (which is stretched on the said line and $f\left(Q_{0}\right)$ ).

Every plane $f(P)$ is contained in a cone $C_{x}^{\prime}$. Therefore, being translated, they are contained in $C^{\prime}$ and, since they cover $V$ with the exception of a plane, so $V \subset C$.

But $V \supset f\left(P_{0}\right)$ and $f\left(P_{0}\right)$ being a maximal plane contained in $\sigma^{\prime}, V=f\left(P_{0}\right)$. This contradicts the supposition.

Thus, $V=f\left(P_{0}\right), f(R)$ is a plane, and the statement 6.5.1 is proved.

6.6. - Now we prove that $f$ is affine.

6.6.1. - $Q$ being a 2-plane containing two generators of $C, f(Q)$ is a 2-plane either.

Proof. - If $Q \subset C$, then $f$ is affine on $Q$ by 6.4. Suppose $Q \not \subset C$ and let $l, l_{1}$ be generators of $C$ contained in $Q$.

Let $P$ be a maximal plane containing $l$ and $R\left(P, l_{1}\right)$ the plane stretched on $P$ and $l_{1}$.

Owing to 6.2.4, the generator $l$ is the intersection of all maximal planes $P$ containing it. To every such $P$ there corresponds the plane $R_{\mathfrak{f}}$ stretehed on $P$ and $l_{1}$. The intersection of all $P$ being $l$, that of all $R_{\mathfrak{f}}$ is the plane $Q \supset l, l_{1}$ :

$$
\cap R_{\mathfrak{P}}=Q
$$

As it is shown in 6.2 .5 , it is possible to transform $l$ into $l_{1}$ by means of reflection in a plane $T$, preserving the cone $C$. Then the plane $P$ is transformed into another maximal plane $P_{1} \supset l_{1}$. The plane $P \cap P_{1}=P \cap T=P_{1} \cap T$ has, in $P$ and $P_{1}$, codimensionality 1 . Hence $R\left(P, l_{1}\right)$ is stretched on $P$ and $P_{1}$, and, owing to 6.5.1, $f(R)$ is a plane.

Now, (3) implies that $f(Q)=\cap f\left(R_{\mathcal{T}}\right)$, and, therefore, $f(Q)$ is a plane, q.e.d.

6.6.2. - It is easy to see that every line $l$ is the intersection of 2-planes $Q$ each of which contains 2 generators of any cone $C_{a}$ with $a \in l$.

The eones $O_{x}$ being equal and parallel to each other, proposition 6.6.1 is applied to every $O_{x}$; therefore $f(Q)$ are 2 -planes and $f(l)$ being their intersection, is a line. This being true for every line $l, f$ is affine.

\section{7. - Proof of Theorem 4, Cases (II), (III).}

7.1. - Let again $A=A_{1} \oplus A_{2}$ and a pseudosealar product be introduced in $A$. Now we consider the cones $K_{a}=\left\{x:(x-a)^{2} \geq 0\right\}$ and $Q_{a}=\grave{K}_{a} \cup(a)$, where $\stackrel{ }{K}_{a}=\left\{x:(x-a)^{2}>0\right\} ; \stackrel{\circ}{K}=\left\{x: x^{2}>0\right\}, Q=\stackrel{\circ}{K} \cup(0)$. 
Let in a space $A^{\prime}$ the cones $K_{b}^{\prime}, Q_{b}^{\prime}$ be analogously defined, and let a one-to-one mapping $f: A \rightarrow A^{\prime}$ be such that for every $a \in A f\left(K_{a}\right)=K_{b}^{\prime}$ or for every $a \in A$ $f\left(Q_{a}\right)=Q_{b}^{\prime}$. Then Theorem $4(\mathrm{II})$, (III) asserts that $f$ is affine and $f(A)=A^{\prime}$.

We shall prove this statement by reducing to the result of $\S 6$, i.e. to Theorem $4(\mathrm{I})$; it will be proved that in both cases $f$ maps the cones $C_{a}:(x-a)^{2}=0$ onto cones $C_{b}^{\prime}:(x-b)^{2}=0$, and, therefore, is affine by the result of $\S 6$.

7.2. - We start with some properties of $K$ and $\stackrel{\circ}{K}$.

7.2.1. - If $a^{2} \geqslant 0$, i.e. $a \in K$, then for every $\lambda \in(0,1)$

$$
K_{\lambda a} \subset K \cup K_{a}, \quad \stackrel{\circ}{K}_{\lambda a} \subset \stackrel{\circ}{K} \cup \stackrel{\circ}{K}_{a}
$$

Proof. - Let $a^{2} \geqslant 0, \lambda \in(0,1)$. The set $K_{\lambda a} \backslash K$ is determined by the inequalities: $(x-\lambda a)^{2} \geqslant 0, x^{2}<0$. Hence, because of $a^{2} \geqslant 0$ and $0<\lambda<1$, we have:

$$
0 \leqslant(x-\lambda a)^{2}=x^{2}-2 \lambda a x+\lambda^{2} a^{2}<\lambda x^{2}-2 \lambda a x+\lambda a^{2},
$$

i.e. $(x-a)^{2} \geq 0$, i.e. $x \in K_{a}$.

Therefore, $K_{\lambda a} \backslash K \subset K_{a}$, i.e. $K_{\lambda_{a}} \subset K \cup K_{a}$.

The set $\stackrel{\circ}{K}_{\lambda a} \backslash \stackrel{\circ}{K}$ is determined by the inequalities $(x-\lambda a)^{2}>0, x^{2} \leqslant 0$ and the same deduction leads to the inclusion $\stackrel{\circ}{K}_{\lambda a} \backslash \stackrel{\circ}{K} \subset \stackrel{\circ}{K}_{a}$, i.e. $\stackrel{\circ}{K}_{\lambda_{a}} \subset \stackrel{\circ}{K} \cup \stackrel{\circ}{K}$.

7.2.2. - If $a^{2}<0$, i.e. $a \notin K$, there exists no such point $b(b \neq a, 0)$ that $\stackrel{\circ}{K}_{b} \subset K \cup K_{a}$, and the more, no such $b$ that $\stackrel{\circ}{K}_{b} \subset \grave{K} \cup \stackrel{ }{K}_{a}$ or $K_{b} \subset K \cup K_{a}$.

Proof. - Let points $a, b$ be given: $a^{2}<0, a \neq b \neq 0$. We shall prove that $\stackrel{\circ}{K}_{b} \not K \cup K_{a}$.

Suppose $b \notin K \cup K_{a}$. Then for any given $o$ and sufficiently small $\lambda,(b+\lambda c) \notin$ $\notin K \cup K_{a}$ : But if $o^{2}>0$, then $(b+\lambda c) \in \stackrel{\circ}{K}_{b}$. Hence $\stackrel{\circ}{K}_{b} \not K \cup K_{a}$ :

Suppose now that $b \in K \cup K_{a}$, e.g. $b \in K$, i.e. $b^{2} \geqslant 0$ (otherwise we can take $a$ for the origin).

Let $b^{2}>0$. Then for any given $o$ and sufficiently small $\lambda$, we have $(\lambda o-b)^{2}>0$, i.e. $\lambda e \in \mathfrak{K}_{b}$. But if we take such 0 that $c^{2}<0$, then $\lambda^{2} e^{2}<0$, i.e. $\lambda c \notin K$. And, since $a^{2}<0$, we have, for small $\lambda$, $(\lambda c-a)^{2}<0$, i.e. $\lambda c \notin K_{a}$. Thus, $\lambda e \notin K \cup K_{a}$, and since $\lambda c \in K_{b}$, so $K_{b} \not K \cup K_{a}$.

Let now $b^{2}=0$, i.e. $b_{1}^{2}=b_{2}^{2}$. Take the vector $c=b_{2}$, i.e. $c_{1}=0, c_{2}=b_{2}$. Then $a^{2}=b c=-b_{2}^{2}$. Using these equalities and $b^{2}=0$, we have

$$
(\lambda c-b)^{2}=-\lambda^{2} b_{2}^{2}+2 \lambda b_{2}^{2}=\lambda(2-\lambda) b_{2}^{2} .
$$

Hence, for $0<\lambda<2,(\lambda c-b)^{2} \geqslant 0$, i.e. $\lambda c \in \stackrel{\circ}{K_{b}}$. 
On the other hand, $c^{2}=-b_{2}^{2}<0$ implies that $\lambda c \notin K$. Besides,

$$
(\lambda e-a)^{2}=-\lambda^{2} b_{2}^{2}+2 \lambda b_{2} a_{2}+a^{2}
$$

and, since $a^{2}<0$, so for small $\lambda,(\lambda c-a)^{2}<0$, i.e. $\lambda c \notin K_{a}$.

Thus, for small $\lambda, \lambda c \in \stackrel{\circ}{K}_{b}$ but $\lambda c \notin K \cup K_{a}$, i.e. $\stackrel{\circ}{K}_{b} \not K \cup K_{a}$, and the statement 7.2 .2 is proved.

7.3. - There take place two following statements in a sense reciprocal to the above statements 7.2.1, 7.2.2.

7.3.1. - If $a^{2} \leqslant 0$, i.e. $a \notin \stackrel{\circ}{K}$, then for every $\lambda \in(0,1)$

$$
K_{\lambda a} \supset K \cap K_{a}, \quad \stackrel{\circ}{K}_{\lambda a} \supset \stackrel{\circ}{K} \cap \stackrel{\circ}{K}_{a} .
$$

7.3.2. - If $a^{2}>0$, i.e. $a \in \stackrel{\circ}{K}$, there exists no such point $b(b \neq a, 0)$ that $K_{b} \supset K \cap K_{a}$ or $\stackrel{\circ}{K}_{b} \supset \stackrel{\circ}{K} \cap \stackrel{\circ}{K}_{a}$.

Proof. - Along with the given pseudosealar product $x y=x, y_{1}-x_{2} y_{2}$, we may consider $\widetilde{x y}=x_{2} y_{2}-x_{1} y_{1}=-x y$, in particular, $\tilde{x}^{2}=-x^{2}$. Respectively, we define the sets $\tilde{K}_{a}: \widetilde{(x-a)^{2}} \geqslant 0, \widetilde{K}_{a}: \widetilde{(x-a)^{2}}>0,\left(\tilde{K}: \tilde{x}^{2} \geqslant 0, \widetilde{K}: \tilde{x}^{2}>0\right)$.

Applying the statements $7.2 .1,7.2 .2$ to these sets, we have $\left(7.2 .1^{*}\right)$. If $\tilde{a}^{2} \geqslant 0$, and $\lambda \in(0,1)$, then $\tilde{K}_{\lambda \alpha} \subset \tilde{K} \cup \tilde{K}_{a}^{\circ}, \tilde{K}_{\lambda \alpha} \subset \tilde{K} \cup \tilde{K}_{a}\left(7.2 .2^{*}\right)$. If $\tilde{a}^{2}<0$, there exist no such points $b(b \neq a, 0)$ that $\mathscr{K}_{b} \subset \widetilde{K} \cup \mathscr{K}_{a}$ or $\widetilde{K}_{b} \subset \widetilde{K} \cup \widetilde{K}_{a}$.

Now we observe the following remarks.

(1) $\tilde{a}^{2} \geqslant 0$ is equivalent to $a^{2} \leqslant 0$, i.e. $a \notin \stackrel{K}{\hbar}$.

(2) $\tilde{a}^{2}<0$ is equivalent to $a^{2}>0$, i.e. $a \in \mathbb{K}$.

(3) $\stackrel{\mathscr{K}}{=}=\left\{x: x^{2}<0\right\}=A \backslash K, \tilde{K}=\left\{x: x^{2} \leqslant 0\right\}=A \backslash \stackrel{i}{K}$.

When the given sets are replaced by their complements, then sums and intersections exchange their roles with the conversion of inclusions. Thus, e.g. $\check{K}_{b} \subset \widetilde{K} \cup \check{\mathscr{K}}_{a}$ is equivalent to $K_{b} \supset K \cap K_{a}$.

Using these remarks we reformulate $\left(7.2 .1^{*}\right)$ and $\left(7.2 .2^{*}\right)$. Then we get just the statements 7.3.1, 7.3.2, and thus they are proved.

7.4. - Now we formulate three immediate corollaries of the above four statements 7.2.1-7.3.2. The cone $K$ can be replaced in them by a cone $K_{0}$. With this change of denotations 7.2 .1 and 7.2 .2 immediately imply:

7.4.1. $-a \in K_{c}, a \neq c$, if and only if there exist such cones $K_{b} \neq K_{a}, K_{c}$ that $K_{b} \subset K_{a} \cup K_{c}$. 
Further, all four propositions 7.2.1-7.3.2, evidently, imply two following statements.

7.4.2. $-a \in K_{o} \backslash \stackrel{\circ}{K}_{c}=C_{c}, a \neq c$ if and only if there exist such cones $K_{b} \neq K_{a}, K_{c}$ that $K_{a} \cap K_{c} \subset K_{b} \subset K_{a} \cup K_{c}$.

7.4.3. $-a \in K_{c} \backslash \stackrel{\circ}{K}{ }_{o}=C_{c}, a \neq c$, if and only if there exist such cones $K_{b} \neq K_{a}, K_{c}$ that

$$
\stackrel{\circ}{K}_{a} \cap \stackrel{\circ}{K}_{c} \subset \stackrel{\circ}{K}_{b} \subset \stackrel{\circ}{K}_{a} \cup \stackrel{\circ}{\boldsymbol{K}}_{v}
$$

7.5. - Now we characterize the vertex of a cone $K_{a}$ in terms of cones $K_{x}$.

7.5.1. - For every point $a,(a)=\cap K_{x}$, where all $K_{x}$ with $x \neq a, x \in K_{a}$ are involved.

Proof. - If $x \in K_{a}$, then $a \in K_{x}$ and therefore $a \in \cap K_{x}$.

On the other hand, for every point $b \neq a$ such $x \in K_{a}, x \neq a$, exist that $b \notin K_{x}$. Hence, $\cap K_{x}=(a)$.

7.5.2. - The vertex $a$ of a cone $K_{a}$ is such a point that $(a)=\cap K_{c}$, where all such $K_{c}$ are involved that there exist cones $K_{b} \subset K_{a} \cup K_{c}, K_{b} \neq K_{a}, K_{c}$.

Proof. - By 7.4.1, the existence of such cones $K_{b}$ is equivalent to $a \in K_{c}$, and thus 7.5.2 follows from 7.5.1.

7.5.3. - Let $f$ be such a mapping $A \rightarrow A^{\prime}$ that for every $x \in A f\left(K_{x}\right)=K_{y}^{\prime}$. Then $f\left(K_{x}\right)=K_{f(x)}^{\prime}$.

Proor. - Let $a$ be a given point and $f\left(K_{a}\right)=K_{d}^{t}$. Then owing to 7.5 .2

$$
f(a)=\cap f\left(K_{x}\right)
$$

where all such $f\left(K_{x}\right) \neq f\left(K_{a}\right)$ are involved that there exist such $f\left(K_{b}\right) \neq f\left(K_{a}\right), f\left(K_{x}\right)$ that $f\left(K_{b}\right) \subset f\left(K_{a}\right) \cup f\left(K_{x}\right)$. The sets $f\left(K_{x}\right)$ being cones $K_{y}^{\prime}$ etc., we conclude that

$$
f(a)=\cap f\left(\boldsymbol{K}_{w}\right) \supset \cap \boldsymbol{K}_{\boldsymbol{v}}^{\prime}
$$

where all such $K_{v}^{\prime} \neq K_{a}^{\prime}$ are involved that there exist $K_{v}^{\prime} \neq K_{b}^{\prime}, K_{v}^{\prime}$ that $K_{v}^{\prime} \subset K_{b}^{\prime} \cup K_{v}^{\prime}$. But applying 7.5 .2 to the cone $K_{b}^{\prime}$, we see that

$$
\cap K_{v}^{\prime}=(d)
$$

Thus, $d=f(a)$, q.e.d. 
7.5.4. $-f(A)=A^{\prime}$ and for every $y \in A^{\prime} f^{-1}\left(K_{y}^{\prime}\right)=K_{f^{-1}(y)}$ i.e. the mapping $f^{-1}$ has the same properties as $f$.

Proof. - Evidently, $A=\cup K_{x}$, where all $K_{x}$ with $x$ belonging to a given cone $K_{a}$ are involved. Hence, using 7.5.3 (and putting $f(x)=y$ )

$$
f(A)=\cup f\left(K_{x}\right)=\cup K_{j(x)}^{z}=\cup K_{y}^{l} .
$$

Since $f\left(K_{a}\right)=K_{f(a)}^{\prime}$, all $K_{v}^{\prime}$ with $y \in K_{f(a)}^{\prime}$ are involved here, and, therefore, $f(A)=A^{\prime}$.

Now $y \in A^{\prime}$ being given there exists $x=f^{-1}(y)$ and, owing to 6.5.2, $f\left(K_{x}\right)=$ $=K_{f(x)}^{\prime}=K_{y}^{\prime}$, i.e. $f^{-1}\left(K_{y}^{\prime}\right)=K_{x}=K_{f^{-1}(y)}$, q.e.d.

7.5.5. - For every a $f\left(C_{a}\right)=C_{f(a)}^{\prime}$.

Proof. - If $x \in C_{a}, 7.4 .2$ implies that there exists such a cone $K_{b} \neq K_{a}, K_{x}$ that

$$
K_{a} \cap K_{x} \subset K_{b} \subset K_{a} \cap K_{x} .
$$

Applying the mapping $f$ and using 7.5.3 (i.e. $f\left(K_{a}\right)=K_{f(a)}^{\prime}$ etc.) we conclude that there exists such $K_{a}^{\prime} \neq K_{f(a)}^{\prime}, K_{f(a)}^{\prime}$ that

$$
K_{f(a)}^{\prime} \cap K_{f(x)}^{\prime} \subset K_{a}^{\prime} \subset K_{f(a)}^{\prime} \cup K_{f(x)}^{\prime}
$$

It means, owing to 7.4.2 applied to the cones $K^{\prime}$, that $f(x) \in O_{f(a)}^{\prime}$. Therefore, $f\left(C_{a}\right) \subset C_{f^{\prime}(a)}^{\prime}$.

But $f^{-1}$ having the same properties as, we conclude that $C_{f(a)}^{\prime} \subset f^{-1}\left(C_{f(a)}^{\prime}\right)=C_{a}$. Therefore, $f\left(C_{a}\right)=C_{f(a)}^{\prime}$, q.e.d.

Thus, the condition $f\left(K_{x}\right)=K_{y}^{\prime}$ implies $f\left(C_{a}\right)=C_{b}^{\prime}$, and the result of $\S 6$ leads to the conclusion that $f$ is an affine mapping of $A$ onto $A^{\prime}$.

7.6. - Now we turn to the cones $Q$.

7.6.1. $-a \in Q_{x}$ if and only if there exist such cones $Q_{b} \neq Q_{a}, Q_{x}$ that $Q_{b} \subset Q_{a} \cup Q_{x}$.

Proof. - We may put $Q_{s}=Q$. Then we have to prove two statements.

(1) If $a \in Q$, then there exist $Q_{b} \subset Q_{a} \cup Q, Q_{b} \neq Q_{a}, Q$.

(2) If $a \notin Q$, no such $Q_{b}$ exist.

The first follows from 7.2.1, if we observe that $Q_{b}=\stackrel{\circ}{K}_{b} \cup(b)$. If $a \in Q, \lambda \in(0,1)$, then 7.2.1 implies that $Q_{p_{a}} \subset Q_{a} \cup Q$.

Let now $a \notin Q$. The proposition 7.2 .2 implies that if $a \notin K$ then no $Q_{b} \neq Q_{a}$, $Q$ is contained in $Q_{a} \cup Q$. 
Suppose $a \in K$, i.e. $a \in K \backslash Q=C \backslash(0)$ and, therefore, $0 \in O_{a} \backslash(a)$. Suppose there is a cone $Q_{b} \subset Q_{a} \cup Q,(b \neq a, 0)$ and let $b \in Q$ (if $b \in Q_{a}$ we take $a$ for the origin). Then $0 \in Q_{b}, b \neq 0$, and, therefore, since $a \in C$, we have $\lambda a \in Q_{b}$ for sufficiently small $|\lambda|$. But, since $a \in O$, so $\lambda a \notin Q_{a} \cup Q$, and therefore $Q_{b} \not Q_{a} \cup Q$.

Thus proposition 7.6.1 is proved.

7.6.2. - Proposition 7.6.1 being quite analogous to 7.4.1, we may apply to the cones $Q$ the same considerations that were applied above to the cones $K$. Thus, by full analogy with 7.5.3, 7.5.4, we shall prove that if for every $x f\left(Q_{x}\right)=Q_{y}^{\prime}$, then $f\left(Q_{x}\right)=Q_{f(x)}^{\prime}$ and the converse mapping $f^{-1}$ has the same properties as $f$.

Then we show that $f\left(C_{x}\right)=C_{f(x)}^{\prime}$, the proof being the same as that of 7.5 .5 with the only difference that 7.4.3 is applied instead of 7.4.2 (we use here that, because of $\left.f\left(Q_{x}\right)=Q_{f(x)}^{\prime}, f\left(\stackrel{o}{K}_{x}\right)=\stackrel{\circ}{K}_{f(x)}^{\prime}\right)$.

Thus, Case (III) of Theorem 4 is reduced to Case (I), and Theorem 4 is completely proved.

7.6.3. - It is more simple to characterize the vertex $a$ of a cone $Q_{a}$ by the following property. A point $z \in Q_{a}$ is the vertex, i.e. $z=a$, if and only if there exist such $Q_{b}, Q_{c}$ that $z \notin Q_{b} \cup Q_{c}$ but $Q_{a} \backslash(z) \subset Q_{b} \cup Q_{c}$ : (The proof is not difficult starting with the observation that for every $b$ with $b^{z}=0$ and $\left.a=-b, Q \backslash(0) \subset Q_{b} \cup Q_{c}\right)$.

This property of the vertex ensures, evidently, that the vertex is mapped into vertex. After that we proceed as it is said above.

\section{REFERENCES}

[1] A. D. Alexandrov, On Lorentz transformations, Uspehi Mat. Nauk, 5, 3 (37) (1950), p. 187 (Russian).

[2] A. D. Alexandrov - V. V. Ovchinnikova, Notes on the foundations of relativity theory, Vestnik Leningrad. Univ., 14 (1953), p. 95 (Russian).

[3] A. D. Almxandrov, The space-time of the theory of relativity, Jubilee of Relativity Theory, Proceedings, Basel, 1956.

[4] A. D. Alexandrov, Philosophical content and meaning of relativity theory, Voprosy filosofii, 4 (1959), p. 67 (Russian).

[5] A. D. Alexandrov, Relativity theory as a theory of absolute space-time, Philosophical problems of modern physics, Moscow, 1959, pp. 269-323 (Russian).

[6] A. D. Alexandrov, A contribution to chronogeometry, Canad. J. Math., 49, No. 6 (1967), pp. 1119-1128.

[7] A. D. Alexandrov, Cones with a transitive group, Doklady Akad. Nauk SSSR, 189, No. 4 (1969), pp. 691-698.

[8] YU. F. BonIsov, On transformations of a pseudoeuctidian space preserving isotopicity of vectors, Izvestija Vysš. Uěebn. Zaved. Matematika, No. 6 (19) (1960), p. 31 (Russian).

[8a] Math. Rev., vol. 25, No. 1480.

[9] A. A. RoBB, A theory of time and space, Cambridge, 1914. 
[10] E. C. Zesman, Causality implies the Lorentz group, J. Math. Phys., 5, No. 4 (1964), p. 490 .

[11] O. S. Rothaus, Order isomorphisms of cones, Proc. Am. Math. Soc., 17, No. 6 (1966).

[12] J. L. Alonso - F. J. Undurian, On the Continuity of Oausal Automorphisms of Spacetime, Communs, Math. Phys., 4, No. 5 (1967), pp. 349-351.

[13] H. Busemann, Time-like spaces, Rospr. mat., No. 53 (1967).

[14] G. Teppati, An Algebraic Analogue of Zeeman's Theorrem, Nuovo Cimento, 54 A, No. 3 (1968), pp. 800-804.

[15] G. Baruccht - G. Teppatr, The Causality Group, Nuovo Cimento, 52 A, No. 1 (1967), pp. $50-61$

[16] G. CeZaR - E. MrHoL, Causal groups of space-time, Communs. Math. Phys., 14, No. 2 (1969), pp. 165-170.

[17] J. Räтz, Zur Definition der Lorentz-transformationen, Math.-phys. Semesterber., 17, No. 2 (1970), pp. 163.167.

[18] H. J. Borchers - G. C. Hegerfeldt, Über ein Problem der Relativitätstheorie: Wann sind Punktabbildungen des $R^{n}$ linear\%, Nachrichten der Akademie der Wissenschaften in Göttingen, II, Math..Phys. Klasse, Jahrgang, 1972, No. 10.

[19] H. J. BoRCHERS - G. C. HEGERFELDT, The structure of space-time transformations, Communs. Math. Phys., 28, No. 3 (1972), pp. 259-266.

[20] A. Gamba - G. Luzzatto, Nuovo Cimento, 33 (1964), p. 1732.

[21] L. MrcheL, Ecole d'ete de Physique Theorique de Oargese (1965).

[22] R. I. Pimenov, Mathematical theory of spaee-time, Leningrad, 1969 (Russian). 